\title{
Breast Conserving Surgery and Sentinel Lymph Node Biopsy under Local Anesthesia for Breast Cancer
}

\author{
Shinichiro Kashiwagi", Naoyoshi Onoda, Tsutomu Takashima, Yuka Asano, Naoki Aomatsu, \\ Masanori Nakamura, Hidemi Kawajiri, Tetsuro Ishikawa, Kosei Hirakawa
}

Department of Surgical Oncology, Osaka City University Graduate School of Medicine, Osaka, Japan.

Email: *spqv9ke9@view.ocn.ne.jp

Received August 21 $1^{\text {st }}, 2012$; revised September 23 ${ }^{\text {rd }}, 2012$; accepted October $8^{\text {th }}, 2012$

\begin{abstract}
Background: Breast conserving surgery and sentinel lymph node biopsy has become the standard operation for early breast cancer. This operation has been performed under local anesthesia for patients that would like short-term admission or for those not indicated for general anesthesia due to complications. This report presents the outcomes of breast conserving surgery and sentinel lymph node biopsy under local anesthesia. Patients and Methods: The study included 61 patients with breast cancer that were all definitely diagnosed before surgery. The indications were preoperatively diagnosed localized DCIS, invasive carcinoma measuring less than $3 \mathrm{~cm}$ in tumor diameter on ultrasound, and tumors with negative axillary lymph nodes. The surgical procedures included breast conserving surgery associated with sentinel lymph node navigation biopsy. Results: The surgery could be performed under local anesthesia in all 61 patients, and no patient was converted to general anesthesia. Four patients had sentinel lymph node metastasis. Surgical stumps were positive in 18 patients $(29.5 \%)$. Ten Gy of boost irradiation of the tumor bed was added to the conventional breast irradiation for these patients. There were no serious complications associated with surgery. Conclusion: Breast conserving surgery and sentinel lymph node biopsy for early breast cancer can be performed safely under local anesthesia. This procedure contributes to shortening the length of hospitalization and thereby saving medical resources without deceasing the quality of treatment.
\end{abstract}

Keywords: Breast Cancer; Local Anesthesia; Breast Conserving Surgery

\section{Introduction}

There is a trend towards more frequently performing breast conserving surgery for breast cancer, and partial breast resection (Bp) combined with sentinel lymph node biopsy (SNB) has became one of the standard treatment methods for early-stage breast cancer [1-5]. The incidence of breast cancer is increasing among Japanese women, as well as in the other countries in the world. The enlightenments for the well-arranged management of breast cancer have been gradually but continuously accepted in public. There is an increasing demand of the integrated and standard systematic treatment in the patients with breast cancer. Therefore, less invasive and less time or cost consuming treatments became necessary and acceptable widely as ever before. Bp combined with SNB has been performed under local anesthesia in the out-patient setting mainly for elderly patients, requesting surgery without admission. Although the technique and significance of SNB under local anesthesia has been reported and discussed enthusiastically, there had been few

*Corresponding author. reports concerning those of performing $\mathrm{Bp}$ at the same time with SNB [6,7]. The advantages of breast conserveing surgery for breast cancer under local anesthesia are avoiding risks due to general anesthesia, and treatment without a hospital stay is therefore possible [6-11]. On the other hand, there remain several critical drawbacks of this procedure, such as the limitations in the pain control during operation and in the extent of resectable areas. This study reviewed the results of breast conserving surgery for breast cancer under local anesthesia in this department, and discussed its feasibility.

\section{Patients and Methods}

This study evaluated a total of 61 patients, with a preoperative diagnosis of breast cancer established based on a core needle biopsy between April 2006 and March 2011. The extent of infiltration of the lesion was evaluated using ultrasonography and MRI. The axillary lymph nodes were evaluated with ultrasonography, CT as well as palpation. The main indication was a diagnosis of ductal carcinoma in situ (DCIS) or invasive carcinoma 
(tumor diameter $<3 \mathrm{~cm}$ ) made preoperatively, without a clinical suspicion of axillary lymph node metastasis. Patients who met these criteria received a full explanation regarding the merits and demerits of systemic and local anesthesia, as well as the risk of recurrence following breast-sparing surgery. Written informed consent was obtained from each patient. Breast conserving surgery for breast cancer was performed under local anesthesia through outpatient visits for those that gave their informed consent. Local anesthesia was conducted with lidocaine $(0.5 \%$ to $1.0 \%)$ containing $5 \mu \mathrm{g} / \mathrm{ml}$ epinephrine (Figure 1). Bp and SNB were performed, and RI and dye were used to identify the sentinel nodes. Tc-99 $\mathrm{m}$ phytate colloid $(1 \mathrm{mCi})$ was injected the day before surgery intracutaneously right above the tumor, subcutaneously, and near the tumor, followed by lymphoscintigraphy to identify the sentinel nodes, explore those in the axilla, and locate sentinel nodes during surgery (Figure 2(a)). Four $\mathrm{ml}$ of ICG solution containing $1.0 \mathrm{ml}$ lidocaine (1\%) was injected subcutaneously through the affected areola of the breast to form a swelling, light massage was applied to the injection site, and a skin incision was then made approximately 10 minutes later to identify the lymph nodes dyed green as the sentinel nodes (Figure 2(b)). A parapapillary $3 \mathrm{~cm}$ arch-wise skin incision was made, a skin flap was created and finally the tumor was excised with a margin of $1 \mathrm{~cm}$ according to its extension. Tumors in the upper lateral quadrant required an approximately $5 \mathrm{~cm}$ axillary incision for both Bp and SNB (Figure 3(a)). Tumors in other locations required an approximately $2 \mathrm{~cm}$ axillary incision for SNB. A half-circle incision around the areola was made for Bp (Figure 3(b)). No drains were placed. A total of $60 \mathrm{~Gy}$ of external radiation was applied to the conserved breast. Patients with positive surgical margins received an additional 10 Gy of boost irradiation (60 Gy in total) instead of additional resection.

\section{Results}

A total 61 cases were surgically treated at this institute.

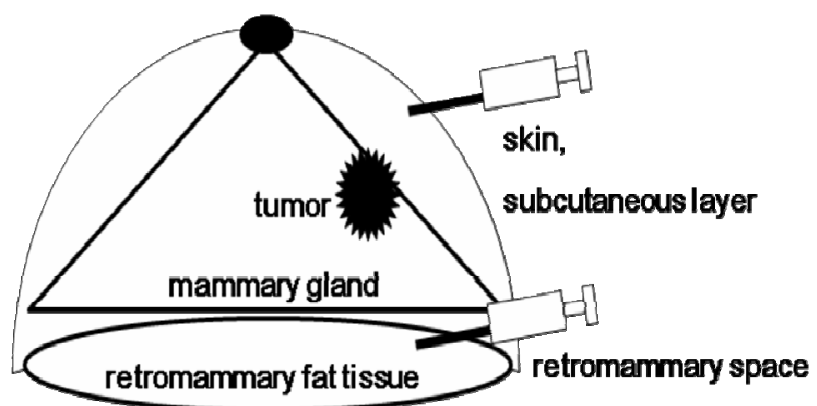

Figure 1. Local anesthesia: Local anesthesia was conducted with lidocaine ( $0.5 \%$ to $1.0 \%)$ containing $5 \mu \mathrm{g} / \mathrm{ml}$ epinephrine.

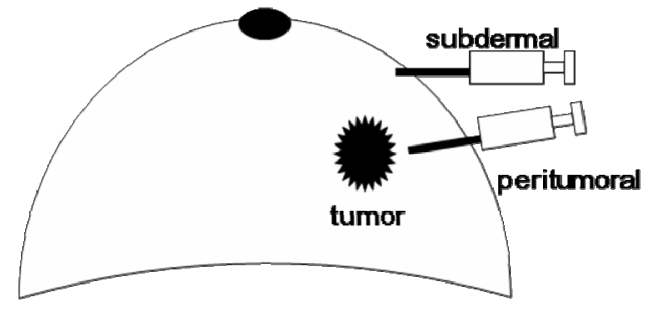

(a)

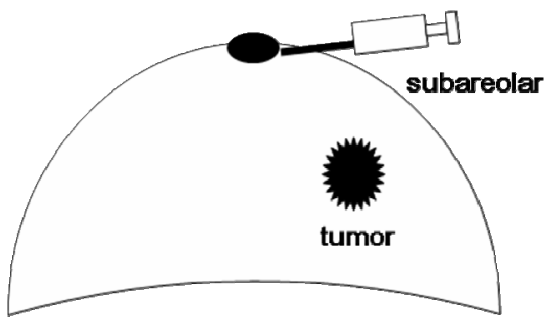

(b)

Figure 2. Sentinel Node Navigation: Radioisotope (99 m Tc phytate): Peritumoral injection one day before surgery (a); Dye (indocyanine green): Intradermal injection to areola (b).

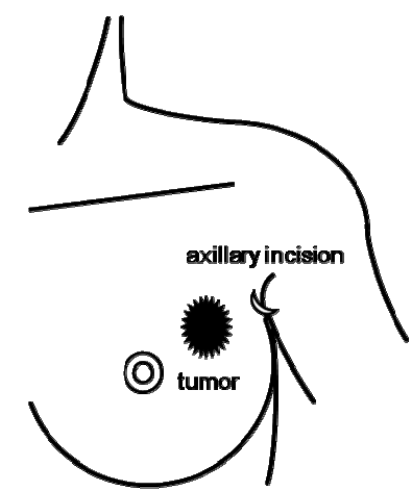

(a)

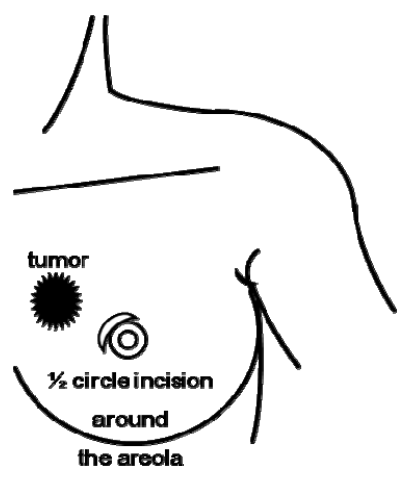

(b)

Figure 3. Surgery: Tumor in upper lateral quadrant: about $5 \mathrm{~cm}$ length axillary incision is made for both Bp and SNB (a); Tumor in the other locations: about $2 \mathrm{~cm}$ axillary incision for SNB. 1/2 circle incision around the areola for $B p$ (b). 
All of the patients underwent surgery with local anesthesia $(100 \%$ of the cases). The average age of the patients was 62 . The tumor stage was: Tis $(\mathrm{n}=5), \mathrm{T} 1(\mathrm{n}=42)$, and T2 $(\mathrm{n}=14)$. The total volume of lidocaine required for surgery was less than $7.0 \mathrm{mg} / \mathrm{kg}$. Surgery could be completed under local anesthesia in all patients, and no patients had to be converted from local to general anesthesia. No hematomas of the resected site, which would require needle drainage, were observed postoperatively. SNB was performed in 44 patients, and metastasis was confirmed in four of them, and all of those patients underwent lymphadenectomy under general anesthesia at a later date. The proportion of patients with positive closed margins in this study $(n=18)$ was equivalent to that previously reported in breast-sparing surgery under general anesthesia (Figure 4). The postoperative observation period ranged from two to 36 months (average: 25 months), and local recurrence developed in four patients, that subsequently underwent breast total resection $(\mathrm{Bt})$ under general anesthesia. No distant organ recurrences were detected. The patients with positive closed margins showed no significant difference in the in the DFS (disease-free survival; Figure 5).

\section{Discussion}

Breast resection under general anesthesia and partial

\begin{tabular}{|c|c|c|}
\hline Day 1 & Day 2 & Day 3 \\
\hline $\begin{array}{c}\text {-Admission } \\
\text {-Visit }\end{array}$ & Day surgery & $\begin{array}{c}\text {-Discharge } \\
\text {-Visit }\end{array}$ \\
\hline $\begin{array}{c}\text {.99mTc injection } \\
\text {-Lymphoscintigraphy }\end{array}$ & Surgery & $\begin{array}{c}\text {-Dressing change } \\
\text {-Radiology consult }\end{array}$ \\
\hline
\end{tabular}

Figure 4. Treatment Schedule: Treatment is undergone 2 nights admission or ambulatory day surgery as patient's preference.

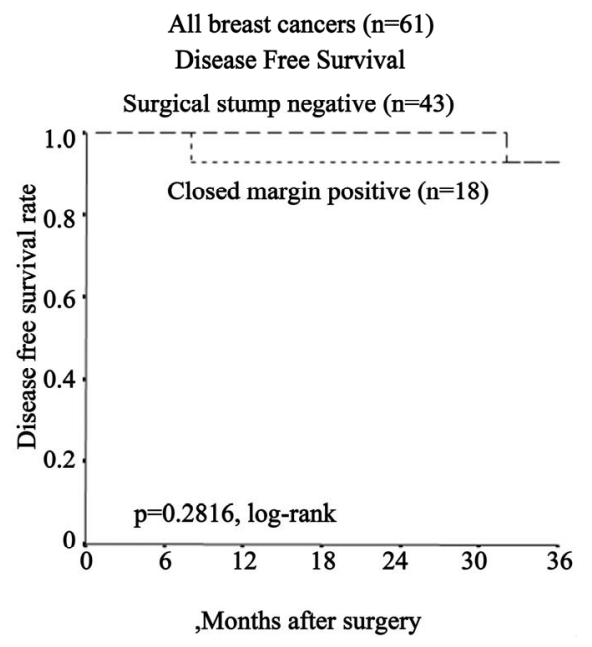

Figure 5. Disease free survival of patients with close margin: Margin status was not concerned with disease free survival. mastectomy combined with axillary lymph node dissecttion has been wildly conducted in the radical treatment of breast cancer. Axillary lymph node dissection under local anesthesia has been reported [8-11], but general anesthesia is considered to be necessary in terms of the efficacy and surgery-associated pain $[12,13]$. SNB, on the other hand, is possible under local anesthesia, and, although the observation periods were short in the present investigation, no recurrences in the axillary lymph nodes were observed. In this study, we could successfuy establish a well-controlled pain relief by adding sufficient volume of local anesthesia to retromammary space under the precise ultrasound guidance before performing Bp. By adding appropriate postoperative radiation therapy after breastconserving surgery, equivalent survival rate was obtained in the patients undergone $\mathrm{Bp}$ in comparison to those undergone mastectomy, and the permissible low-rate of recurrence in the residual-breast could also be obtained $[1,2]$. In addition, according to the well-known results of the NSABP B-32 large-scale clinical trial, demonstrating that completion surgical treatment by axillary lymph node dissection did not affect the long-term prognosis of the cases with negative SNB result, there is a growing trend to omit axillary lymph node dissection in patients with pathologically confirmed negative SNB result [14]. A recent study, ACOSOG-Z0011, demonstrated the possibility to omit salvage axillary dissection in certain population of the cases with positive SNB under several conditions by adding adjuvant therapies [15]. Further evidences for the meaning to perform SNB will be revealed in the near future. Currently, there is no doubt performing SNB to obtain the accurate staging of the patients with early breast cancer for conducting integrated treatment. In other words, less invasive surgical techniques can be applied in order to obtain the specimens to confirm the pathological factors to establish an individual treatment strategy. The extent of infiltration of breast cancer tor partial mastectomy should be accurately determined before surgery, in order to decrease the number of positive closed margins. Ultrasonography and MRI are useful [16-19], and the resection range is determined based on these tests. The rate of positive closed margins in the present study was equivalent to that obtained in a previous study that investtigated breast-sparing surgery under general anesthesia [20]. The patients with positive surgical margins underwent boost irradiation instead of additional resection, but no differences in the DFS were seen, suggesting that boost irradiation is effective. The tolerability of boost irradiation was confirmed, because no complications were observed either during or after surgery.

\section{Conclusion}

It is necessary to accumulate further cases of breast- 
sparing surgery under local anesthesia, in order to establish the precise criteria, and determine the efficacy as well as tolerability. However, the current results suggested that the above described surgical procedure is useful as one type of minimally-invasive surgery.

\section{REFERENCES}

[1] U. Veronesi, et al., "Twenty-Year Follow-Up of a Randomized Study Comparing Breast-Conserving Surgery with Radical Mastectomy for Early Breast Cancer," The New England Journal of Medicine, Vol. 347, No. 16, 2002, pp. 1227-1232. doi:10.1056/NEJMoa020989

[2] B. Fisher, et al., "Twenty-Year Follow-Up of a Randomized Trial Comparing Total Mastectomy, Lumpectomy, and Lumpectomy Plus Irradiation for the Treatment of Invasive Breast Cancer," The New England Journal of Medicine, Vol. 347, No. 16, 2002, pp. 1233-1241. doi:10.1056/NEJMoa022152

[3] G. F. Schwartz, et al., "Proceedings of the Consensus Conference on the Role of Sentinel Lymph Node Biopsy in Carcinoma of the Breast April 19 to 22, 2001, Philadelphia, Pennsylvania," Human Pathology, Vol. 33, No. 6, 2002, pp. 579-589.

doi:10.1053/hupa.2002.124117

[4] U. Veronesi, et al., "A Randomized Comparison of Sentinel-Node Biopsy with Routine Axillary Dissection in Breast Cancer," The New England Journal of Medicine, Vol. 349, No. 6, 2003, pp. 546-553. doi:10.1056/NEJMoa012782

[5] U. Veronesi, et al., "Sentinel-Lymph-Node Biopsy as a Staging Procedure in Breast Cancer: Update of a Randomised Controlled Study," Lancet Oncology, Vol. 7, No. 12, 2006, pp. 983-990. doi:10.1016/S1470-2045(06)70947-0

[6] A. Y. Joseph, et al., "Simple Anesthesia for Simple Mastectomies," Breast Cancer Research and Treatment, Vol. 77, No. 2, 2003, pp. 189-191. doi:10.1023/A:1021386722363

[7] G. W. Carlson , "Total Mastectomy under Local Anesthesia: The Tumescent Technique," Breast Journal, Vol. 11 , No. 2, pp. 100-102. doi:10.1111/j.1075-122X.2005.21536.x

[8] P. Fenaroli, et al., "Axillary Sentinel Node Biopsy under Local Anaesthesia in Early Breast Cancer," Annals of Oncology, Vol. 11, No. 12, 2000, pp. 1617-1618. doi:10.1093/oxfordjournals.annonc.a010407

[9] A. Luini, et al., "Sentinel Lymph Node Biopsy Performed with Local Anesthesia in Patients with Early-Stage Breast Carcinoma," Annals of Surgery, Vol. 137, No. 10, 2002, pp. 1157-1160.

[10] M. L. Smidt, et al., "Sentinel Lymph Node Biopsy Performed under Local Anesthesia Is Feasible," American
Journal of Surgery, Vol. 187, No. 6, 2004, pp. 684-687. doi:10.1016/j.amjsurg.2003.09.009

[11] A. Luini, et al., "The Sentinel Node Biopsy under Local Anesthesia in Breast Cancer: Advantages and Problems, How the Technique Influenced the Activity of a Breast Surgery Department; Update from the European Institute of Oncology with More than 1000 Cases," Breast, Vol. 16, No. 5, 2007, pp. 527-532. doi:10.1016/j.breast.2007.04.003

[12] C. L. van Berlo, et al., "Ambulatory Sentinel Node Biopsy under Local Anaesthesia for Patients with Early Breast Cancer," European Journal of Surgical Oncology, Vol. 29, No. 4, pp. 383-385. doi:10.1053/ejso.2002.1420

[13] N. Athey, et al., "Day-Case Breast Cancer Axillary Surgery," Annals of The Royal College of Surgeons of England, Vol. 87, No. 2, 2005, pp. 96-98. doi:10.1308/1478708051667

[14] D. N. Krag, et al., "Technical Outcomes of SentinelLymph-Node Resection and Conventional AxillaryLymph-Node Dissection in Patients with Clinically NodeNegative Breast Cancer: Results from the NSABP B-32 Randomised Phase III Trial," The Lancet Oncology, Vol. 8, No. 10,2007 , pp. 881-888. doi:10.1016/S1470-2045(07)70278-4

[15] A. E. Giuliano, The Lancet Oncology Axillary Dissection vs No Axillary Dissection in Women with Invasive Breast Cancer and Sentinel Node Metastasis: A Randomized Clinical Trial," Journal of the American Medical Association, Vol. 305, No. 6, 2011, pp. 569-575. doi:10.1001/jama.2011.90

[16] C. A. Finlayson and T. A. MacDermott, "Ultrasound Can Estimate the Pathologic Size of Infiltrating Ductal Carcinoma," Annals of Surgery, Vol. 135, No. 2, 2000, pp. 158-159.

[17] S. Akashi-Tanaka, et al., "Diagnostic Value of ContrastEnhanced Computed Tomography for Diagnosing the Intraductal Component of Breast Cancer," Breast Cancer Research and Treatment, Vol. 49, No. 1, 1998, pp. 79-86. doi:10.1023/A:1005986529910

[18] L. Esserman, et al., "Utility of Magnetic Resonance Imaging in the Management of Breast Cancer: Evidence for Improved Preoperative Staging," Journal of Clinical Oncology, Vol. 17, No. 1, 1999, pp. 110-119.

[19] G. Amano, et al., "Correlation of Three-Dimensional Magnetic Resonance Imaging with Precise Histopathological Map Concerning Carcinoma Extension in the Breast," Breast Cancer Research and Treatment, Vol. 60, No. 1, 2000, pp. 43-55. doi:10.1023/A:1006342711426

[20] Y. Komoike, et al., "Analysis of Ipsilateral Breast Tumor Recurrences after Breast-Conserving Treatment Based on the Classification of True Recurrences and New Primary Tumors," Breast Cancer, Vol. 12, No. 2, 2005, pp. 104111. doi: $10.2325 / \mathrm{jbcs} .12 .104$ 